\title{
The components of a digital asset management system
}

\begin{abstract}
David Austerberry
has worked in the world of asset management since 1997. He was product manager for Pro-Bel's television playout automation and media asset management product lines. The next step was into systems integration for a content management service provider utilizing streaming media technologies. He has written two books: Digital Asset Management (Focal Press, 2004) and The Technology of Video and Audio Streaming, 2nd edition (Focal Press, 2004). He has presented papers on interactive television and digital asset management at many conferences including the National Association of Broadcasters Convention and the International Broadcasting Convention. He is now a director of Informed Sauce, a provider of training services to the television industry, and Editor of Broadcast Engineering world edition.
\end{abstract}

Keywords: software architecture, modular approach, multi-tier model, object-oriented, thirdparty applications, scalable

Abstract This paper looks at the components of digital asset management (DAM). Two views are taken of the system, one is the software architecture and the other is content-centric. The evolution of software architectures is covered from the days of mainframe computers to the multi-tiered systems used by DAM. The components of asset management systems are detailed, followed by an overview of the distributed software systems used to deploy these systems. This paper is based on one of the introductory chapters of the author's book Digital Asset Management (Focal Press, 2004).

\section{INTRODUCTION}

A DAM system uses a suite of applications linked together to provide a seamless experience to the user. It is not just a piece of shrinkwrapped software that can be used straight out of the box. Rather, it is a collection of many components that have been carefully selected to meet the needs and budgets of the enterprise.

The system should also have links to existing software applications: accounts, customer records, and planning systems. To maximize the operational benefits, it is advantageous for these applications to exchange data with the DAM system. Like any enterprise-wide deployment of software, much of the installation costs will be for the professional services. These are usually required to merge the many stand-alone applications into a seamless operating environment, and to customize the business logic to suit the needs of the customer.

This list details some of the components that may be found in a typical installation:

- content repository;

- digital asset management application;

- databases;
- search engine;

- indexing workstations;

- rights management application;

- web portal.

The complete DAM system is rarely the product of a single vendor. Most partner with specialists for services like the search engine, video logging, speech recognition and the underlying database management system. The core DAM acts like glue, it binds all these functions together using the business rules. The users see a unitary view of all the systems which is created by the presentation layer.

This modular approach allows the best-ofbreed solutions to be used, and should retain flexibility for the customer to stick with their favored databases and operating platforms. Clearly with all these different products, a corporation will need a prime contractor (usually the systems integrator) to ensure that ultimately, the solution operates satisfactorily as a whole and meets the original, agreed requirements.

The system architecture can be viewed in a number of ways. One view is that of the software system architecture. This is usually 
multi-tiered, with presentation, business and data layers. Another view is content-centric, with the system in shells around the core assets and modifying the view the user sees.

One of the primary functions of any DAM system is to relieve the users of the need to perform repetitive tasks. This frees them to concentrate on the more creative side of media authoring and publishing. The design of the user interface (UI) is key to the success of the product. The other issue of great importance to the user is the way that the system fits with the existing corporate processes. If too many changes are made to the ways of working, the users can feel alienated from the asset management. Limited scale trials are very useful for the shakedown of workflow issues. The goal is for the users to feel excited by the opportunities presented by the system, not to resent what could be viewed as a system imposed to reduce manning levels. The goal is a win-win for the corporate management and the knowledge workers.

\section{MAINFRAMES TO MULTI-TIER}

Looking back through the history of enterprise computing, it all started with the mainframe and simple clients based upon the video terminal or visual display unit (VDU). Smaller mainframes (the minicomputer) were developed to suit the needs of medium-sized businesses, and then IBM launched the personal computer (PC). This allowed even the smallest business to produce professional-looking correspondence and to run accounts software.

As the power of the PC increased, it became feasible to run graphics-rich programs. Although the early PCs had clunky text interfaces with boxy graphics, Apple pioneered the way with mouse-driven windows. These led to the WYSIWYG (what you see is what you get) UI that we all demand today.

As the costs of the desktop PC dropped to little more than the desks they sat on, business users demanded the same UI as the personal computer. A good example is the word processor. The first products used the mainframe computer to run the application. To see the final layout, the operator had to print out a copy. To produce a complex layout was an iterative process involving many intermediate printouts. The WYSIWYG word processor application did away with this, dramatically improving productivity. At the same time, it enabled the user to be more creative with their layout. This ability to offer new possibilities extends to asset management.

After the replacement of the video terminals with PCs, the minicomputer that ran the wordprocessing became redundant; the central file server replaced it.

\section{Client-server}

This new architecture was called client-server. The user now had the power of the new office application suites. The combination of word processing, spreadsheets and basic drawing facilities met most of the average office worker's needs. Alongside this, the old mainframe systems still ran the company databases for online transaction processing, the sales order processing, inventory control and manufacturing resource planning - everything that makes the enterprise tick.

Some asset management products adopted the client-server approach. The client includes the business logic and a presentation layer for the UI. The server is essentially just a relational database. The clients interact with each other through the database management system (DBMS) using stored procedures and triggers. Client-server is fine for small systems, but in practice, it does not scale well beyond 100 clients.

Initially everybody was happy with the wonders of modern computers throughout the business. However, it soon became apparent that running thousands of PCs, with thousands of instances of the applications, was very expensive. First, there were the costs of the per-seat licensing. Then there were the costs involved in updating machines when new software releases came out (all too frequently). But the real killer was the ease with which the average office worker could reduce his or her own computer to a state of malfunction. Either by misconfiguring the applications, or by illicitly loading unofficial software, the machine would grind to a halt, ready for rescue by the IT department.

The cost of all this downtime, and the staffing levels required in IT, began to be of great concern to senior management. There had to be better ways. 


\section{MAINFRAME}

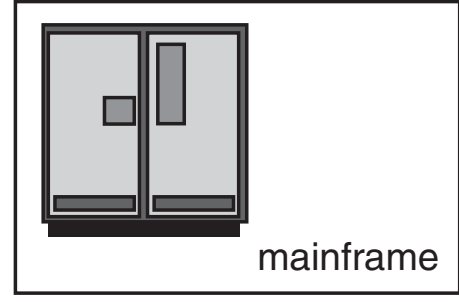

\section{CLIENT/SERVER}

transaction processing word processing
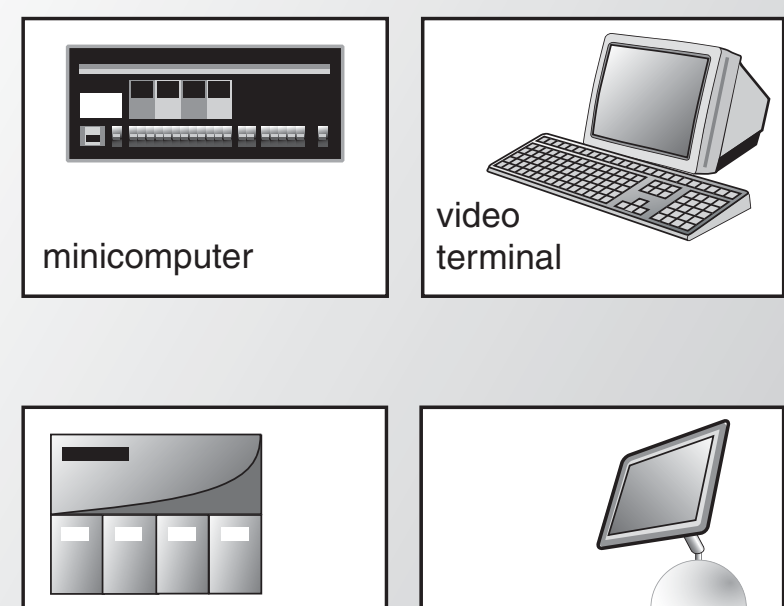

file server/ database

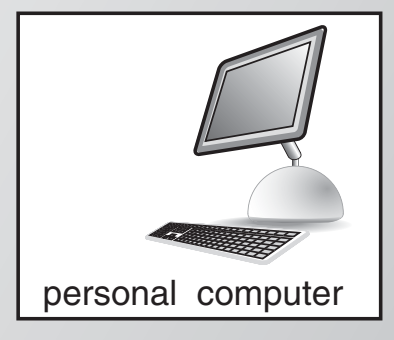

personal computer transaction processing word processing video terminal

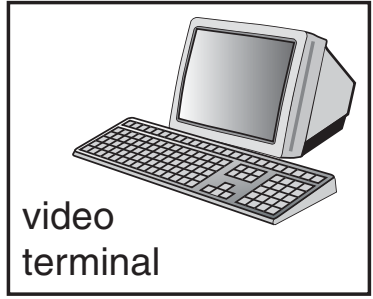

\section{WORKSTATION} graphics compositing 3-D modeling video graphics

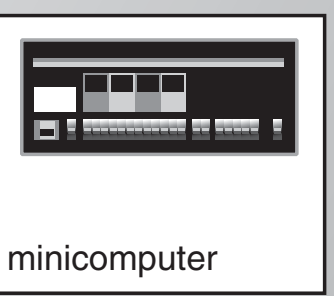

minicomputer

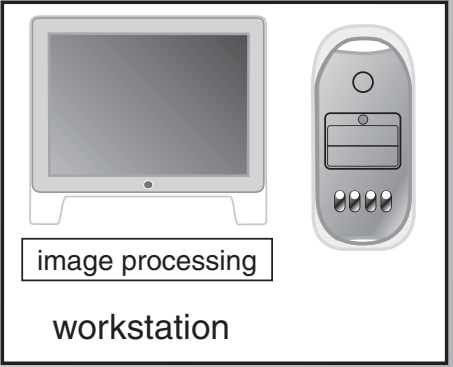

\section{MULTI-TIER COMPUTING}
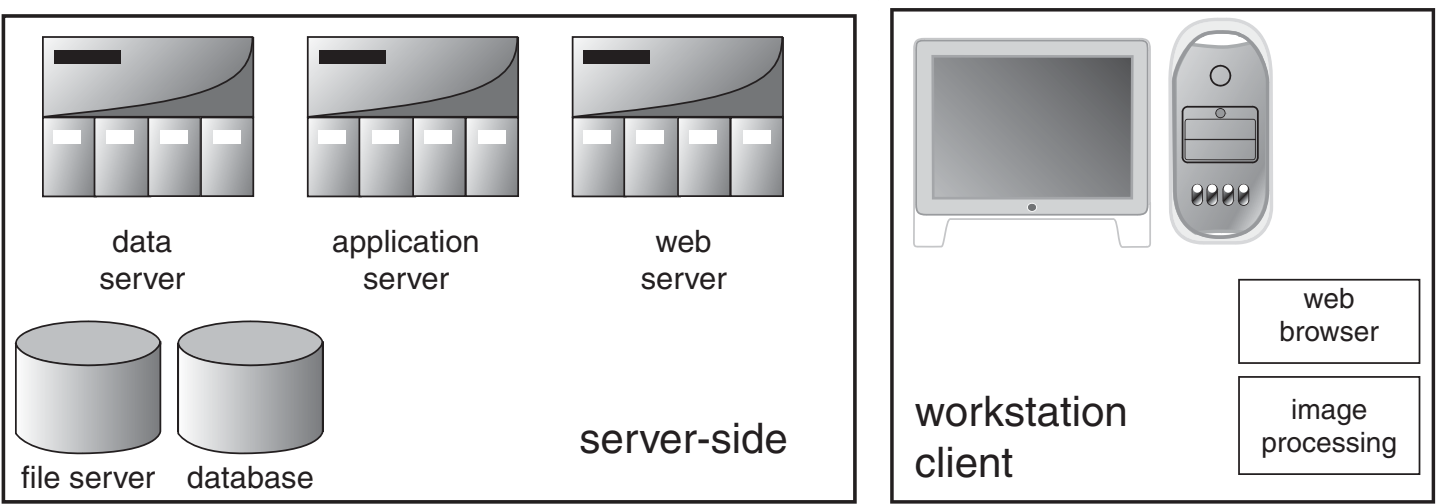

Figure 1: The evolution of multi-tier computing 


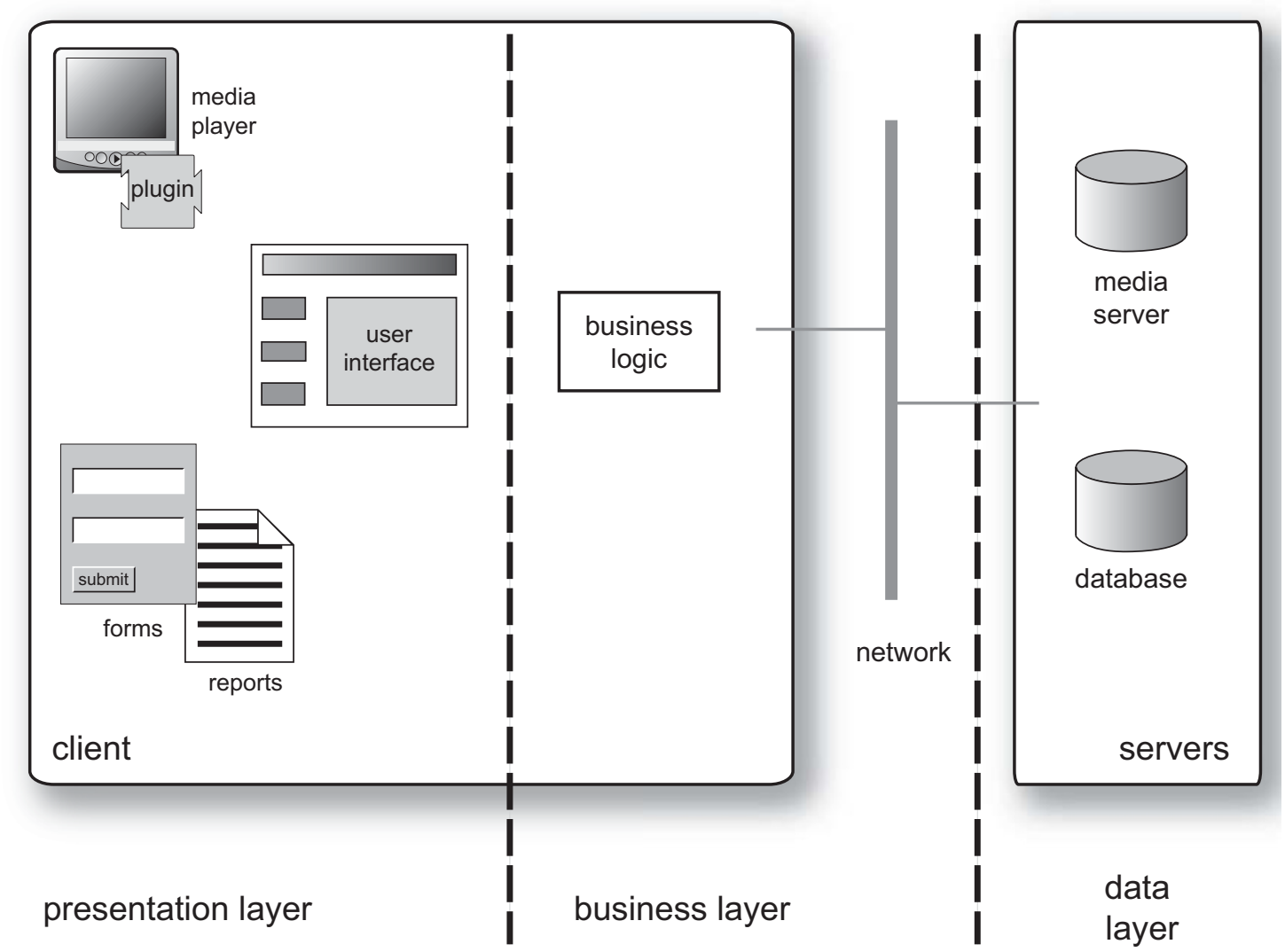

Figure 2: The client-server model

One approach was to use the 'network' PC. This had no removable storage; no CD or floppy drives, so it was difficult to load unauthorized software. Modern operating systems allow remote access by a system administrator, so that software updates could be performed from a central point over the corporate network. This is fine in a controlled world, but what about the marketing communications department? How do they exchange files with external designers? How do personnel staff backup confidential information? Most enterprises accept that distributed applications are not going away. The network PC has a place, but it is not the answer for the typical distributed asset management application.

IT professionals have taken two paths to counteract these problems. One path is to retain the advantages of the client-server environment; the other is the thin client. The solution in the client-server environment is to use a desktop management system. This allows the IT managers to have complete control over software distribution to the clients. The operating system access is configured to forbid local software installation and configuration. Applications, patches and updates are all loaded from a central point. The system also offers remote troubleshooting, and can usually self-heal local software faults. Such a system removes most of the problematic maintenance issues that have dogged client-server and distributed installations.

\section{The thin client}

The other route is the thin client. This could be seen as a return to the simple terminal of the old mainframes. Today's user has many expectations from a UI that the VT100-style terminals could not offer. Perhaps the most important are support for graphics and mouse-driven user interaction.

The current implementation of a thin client is to use a web browser on a standard PC. One 
option is to add additional functionality to the web environment by adding a Java virtual machine. This supports applets to run small programs on the client. The business logic is then transferred from the client to a third tier, the application server.

The two paths are best suited to different applications. The client-server is ideal for office applications, where the work is file-based. The user loads a file from a central server, edits it locally, and then saves back to the server. The user has all the advantages of a powerful application running locally. Imagine the latencies of using a spell checker while typing, if the word processor were running on a remote server.

Data-record oriented applications are more suited to the central data server. Information is viewed and updated from the client, but the data resides on the central server.

DAM has elements of both. There is much data processing, relating to metadata queries and updates. There is also file processing, the creative processes applied to the content: editing, format conversion and compositing.
The outcome is a hybrid approach. Many of the productivity tools run locally on the client machines. They can be centrally maintained using a desktop management system. These include Word, Excel, Quark and PhotoShop. The craft-oriented applications like the nonlinear editor may still run stand-alone applications (eg Avid, Final Cut Pro and Premier Pro) but will be maintained locally at the workstation. The database intensive applications, with workflow management and peer-to-peer collaboration, can use a web browser to view a presentation layer in the application server.

\section{Three tier}

The complex business logic of asset management leads naturally to a central application server.

This can support the collaborative workflow and communications between the users' workstations with central business logic to manage communication between the software clients. This logic forms a third layer between the database and the client.

Many enterprises planning to purchase DAM
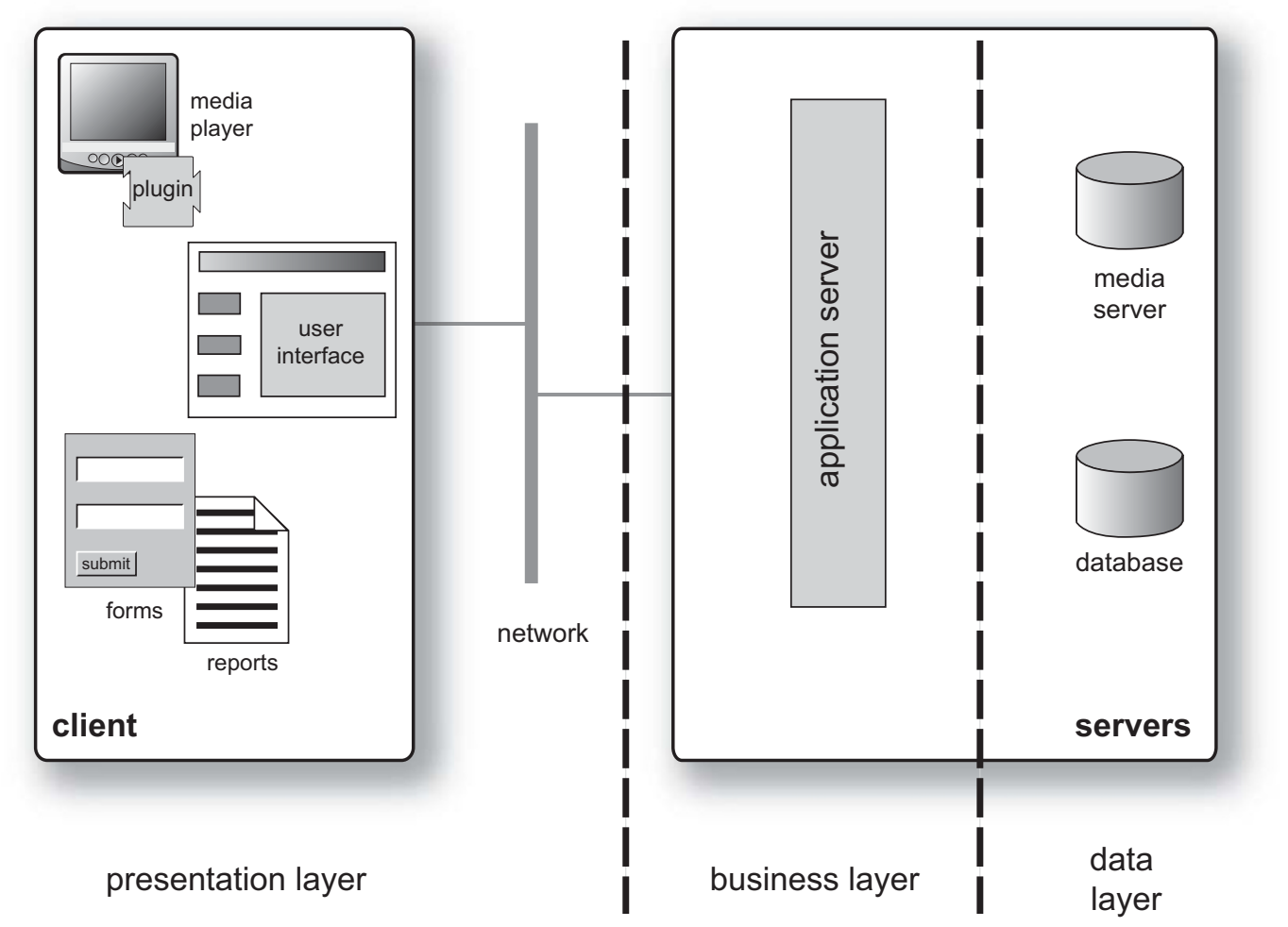

Figure 3: The three-tier model 
systems will want to minimize the cost of each client seat. Many users only want to search, then preview files. Such an application does not warrant the cost of a fully-featured client running a local application. The enterprise may well want to share asset information with suppliers and customers.

The requirement to minimize cost dictates a thin client, especially for remote users. Suppliers of creative services may well use Apple Macs. Other partners may use Linux. An enterprise may use Windows for office applications and UNIX for transaction processing. All this demands a client that is agnostic to operating systems, and that can use widely available communications protocols. Well there is one such client - the web browser.

Rather than running the two tiers of the client-server model, the web browser requires at least three tiers with a web server generating the presentation.

\section{Multi-tier}

The multiple or n-tiered approach to computing splits an application into manageable chunks. Typically, there is a data layer, a presentation layer, and a layer of business logic linking the two. This has many advantages for the IT

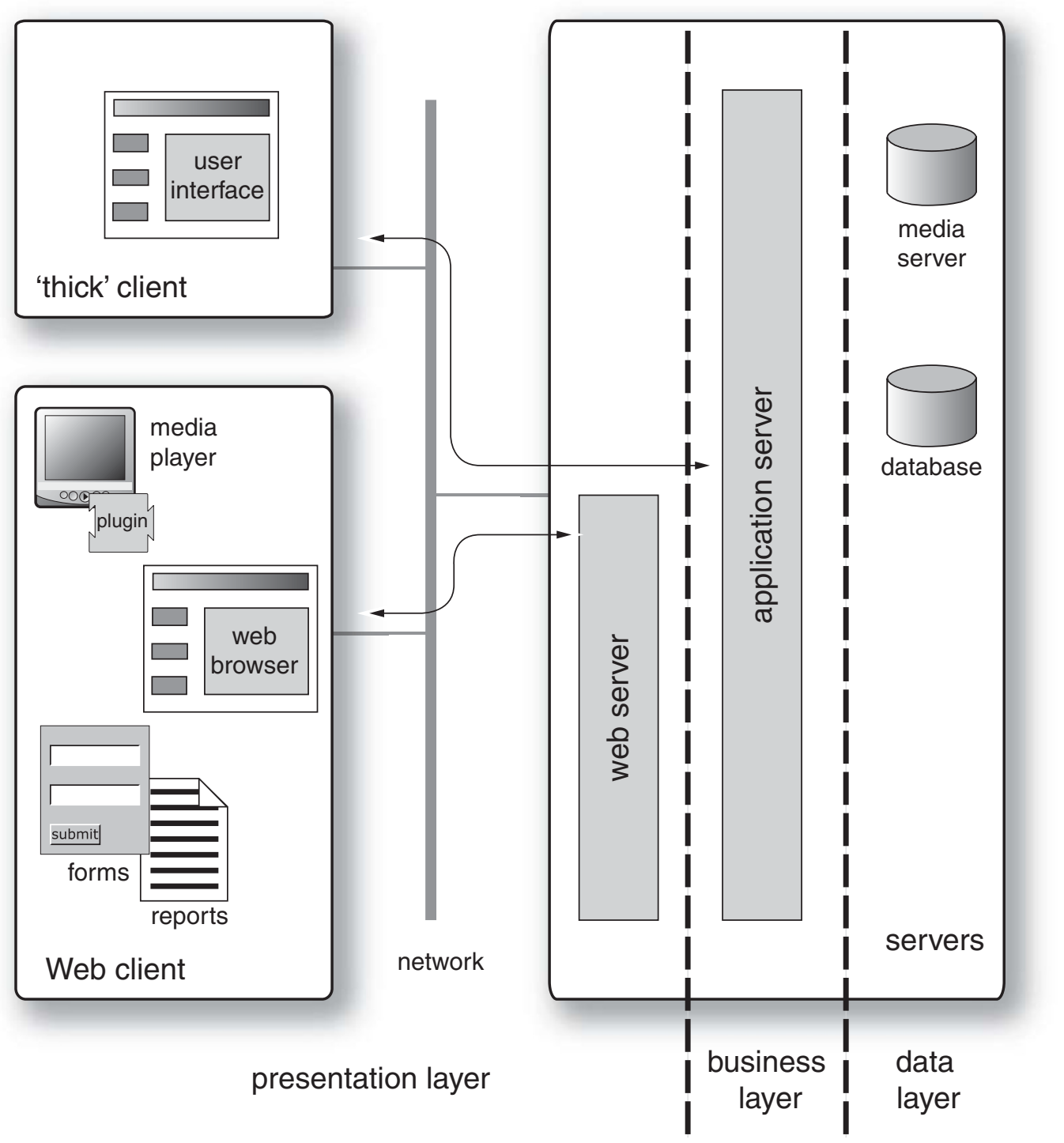

Figure 4: Multi- or n-tier computing 
department. The data layer can be a standard relational database; DB2, Oracle, SQL Server, it does not matter. These are mature products, with predictable behavior, and formal maintenance procedures. The presentation layer is just a web server, again no great problem to operate and maintain. With this architecture the DAM can be broken away into a separate box called the business logic. Here the rules for manipulating the data, and the connections to third-party applications, can all reside on a separate application server that runs the core asset management software.

The central server has now become fragmented into functional blocks: data server, web server and application server.

\section{Application server}

The business logic runs on the application server. This provides the services and framework to run the software objects that implement the business logic. The framework has two sub-systems. One is to implement the rules of the business logic, the processes like the workflow and categorization. The second is the transaction management for updating metadata records in the database.

\section{The data tier}

The data tier is usually a relational database, although this is for administrative convenience rather than elegance of design. It could alternatively be an object database. The DBMS controls the connections for the general maintenance of the database. The application server is a client to the database. The DBMS manages the transactions to enter and update data, and the information retrieval to generate views and reports of the data. In addition, the database administrator will have a number of tools to maintain the database.

Originally, each database product required a different driver for the applications to perform operations on the data. Microsoft developed the language-independent Open Database Connectivity (ODBC) interface that rationalized the database connections into a single standard. This was later joined by Java DataBase Connectivity (JDBC), which exposes a Java application program interface to the database drivers. These technologies have much simplified the connection between the business logic and the database. There are limitations to ODBC; it was designed to use structured query language (SQL) for access to relational

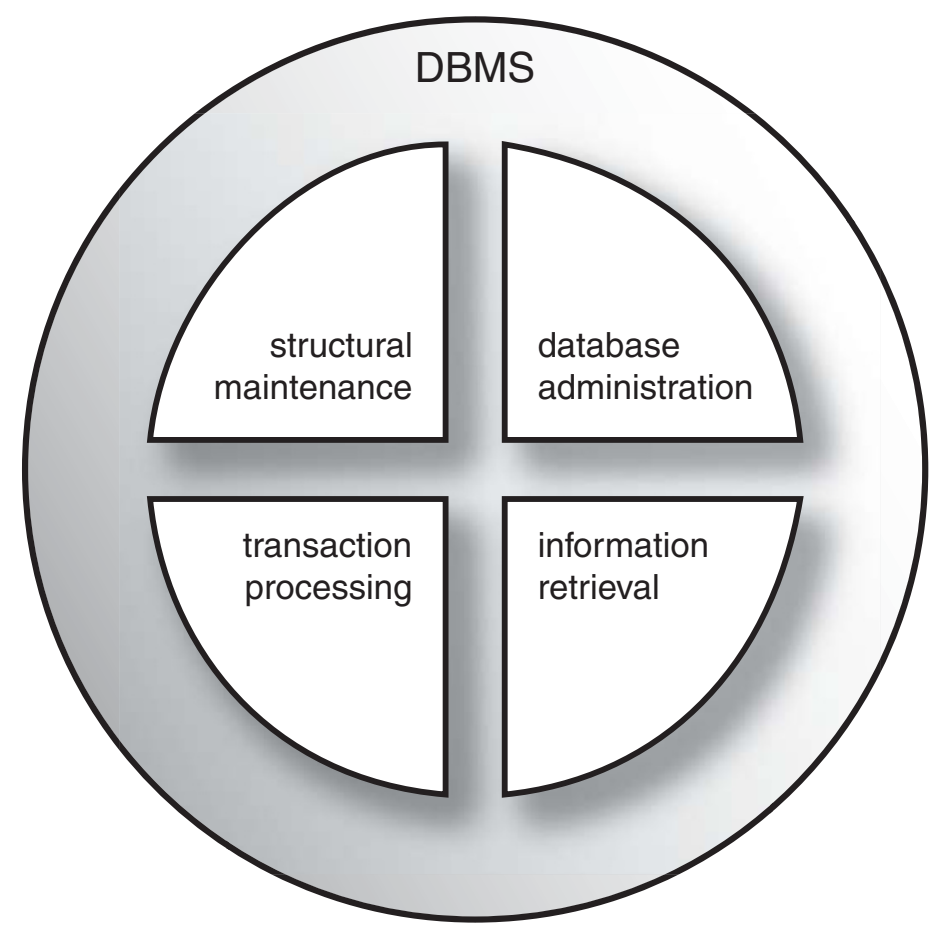

Figure 5: Elements of the database management 
databases. There are now many data sources that are not relational - mail systems, object databases, file servers. There have been newer and more efficient components that can provide a more universal data access than ODBC.

\section{Limitations of the web tier}

Some clients need more functionality than can be provided by the basic web browser. For example, media ingest requires PCs equipped with specialist video encoding cards and extra processing power for the speech analysis. Many DAM products use a hybrid approach. Simple search and retrieve operations can be made from a web browser, but more complex ingest and editing operations use a full client application.
So far, this paper has described closed systems - a corporate network. Most collaboration in asset management extends outside the enterprise. Customers and partners will need controlled access to the asset management. Again, the web browser provides a simple option. The remote user needs no special software application; the technology is familiar and ubiquitous.

\section{THE CONTENT MODEL}

The Society of Motion Picture and Television Engineers/European Broadcasting Union Task Force on Harmonized Standards (1998) developed a system model to represent the relationships between signals, processes and control systems. This report has become a very

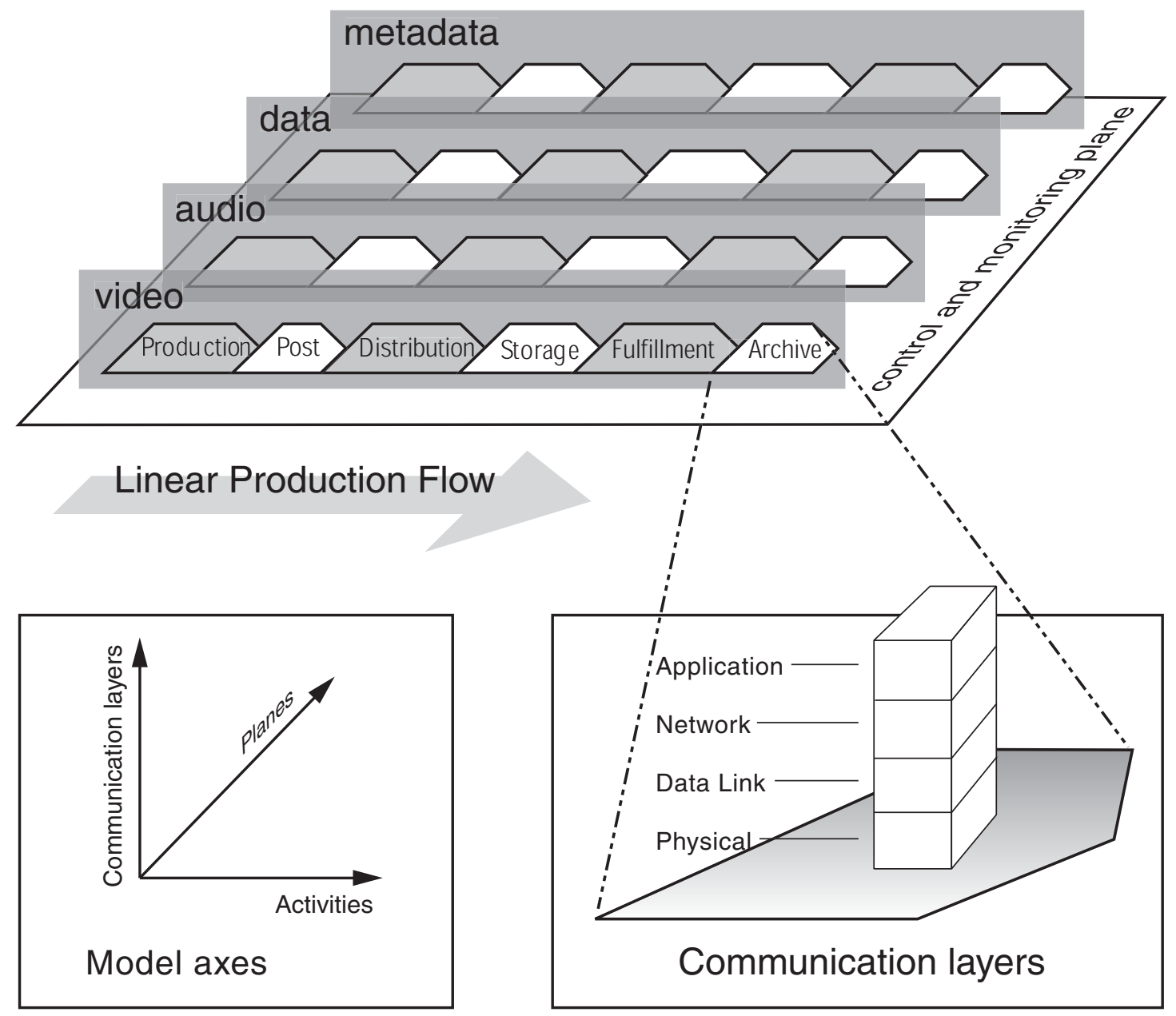

Figure 6: System model for television production 
popular starting point for building object models of content.

The model has three orthogonal axes: activities, content planes and communication layers. The communication layers represent the intercommunication between peer entities. The layers are similar to the ISO open systems interface: application, network, data link and physical. A control and monitoring plane underlies the planes for activities and content.

This model focused on television content, so splits content into audio, video and data (known as essence) and metadata, but the principle could be applied to other forms of content. Essence is the raw content that represents the pictures, sound and text that are delivered to the user. Data essence can be graphics, animation, text files and still images. An example of data essence is the closed caption.

The activities are those typical of the processes in the workflow of television production. The production phase represents planning and shooting. Post is the post-production, where the original footage is edited into a final program, the graphics are added and the sound design implemented. Distribution is the dissemination of the finished program to the publishers. These could be broadcasters or DVD distributors. The storage operation is optional, as the product may be aired immediately. Fulfillment is the delivery to the consumer. This could be as broadcast television transmission, or as sell-through media: DVD and VHS. Finally, the program is archived to a vault.

This flow is linear, but many programs are brought out of the vault and returned to the post stage for repurposing and reuse in a cyclical process. We see more of this today to meet the demands of multi-channel broadcasting plus new media distribution channels.

\section{THE ASSET \\ MANAGEMENT SYSTEM}

The asset management can be looked at as a number of blocks. This view is somewhat flexible, what is content, what is data? In this view, content that is stored in a file system (like Network File System) is called data, content that is stored as video on tape or in special video servers is treated separately as content and managed through the media management component.

Most products are modular, and sit like a hub at the center of media operations and processes. The core asset management should offer a number of different functions:

- indexing and categorization;

- search;

- content editing;

- workflow management;

- task and project management;

- resource management;

- user management;

- $\quad$ storage management.

The asset management functions will be supported by a number of services:

- load balancing and fault tolerance;

- access control;

- security;

- configuration.

\section{Indexing and categorization}

The index module uses metadata generated at the ingest stage to create an index and catalog that can be used by the search engine. The indexing can be fairly simple using a few key fields: file name, tile, author, and keywords. For a large repository, a more sophisticated index will be required. This will avoid the common problem of huge result sets with little relevance to the search criteria. In order to return smaller and more relevant result sets one technique is to use concepts rather than keywords.

\section{Search}

Search is an essential facility for any asset management. The user can use the search engine to find content within the repository. Search engines vary from a basic keyword search through to natural language search. Concept searches may well suggest allied content that may be relevant.

\section{Content editing}

Many asset management applications include basic video editing facilities. A user can assemble a number of scenes or clips into a contiguous sequence. This assembly of clips generates an edit decision list (EDL). This list can be used later with a non-linear editor to conform the source media at broadcast resolution to finished video content. 


\section{Workflow management}

One of the big advantages of asset management is the workflow management. The system provides a platform to automate the business processes. It should have basic features like email alerts. It may include more comprehensive facilities. These can help the project team collaborate and share content.

\section{Task and project management}

Much of the use for DAM is for projects to create or repurpose content. A module for task management can aid the smooth running of a project.

\section{Resource management}

Facilities like video ingest will usually have a limited number of encoding ports and associated videotape recorders. A resource management module can manage the allocation of such hardware to a prepared schedule. There may also be the need to manage human resources, like the tape operators.

\section{User management}

This module manages the users of the system, with facilities to personalize the web interface, associate with projects, and modify personal configuration settings.

\section{Storage management}

The asset management application should offer a number of services to support the storage management. These will include storage networks, hierarchical storage management to manage the disk, near-line and off-line, and media management for removable and analog storage.

\section{System services}

The core services of asset management can vary from platform to platform but certain elements will be essential.

\section{Load balancing and fault tolerance}

Much like a mainframe computer, the application server that runs the asset

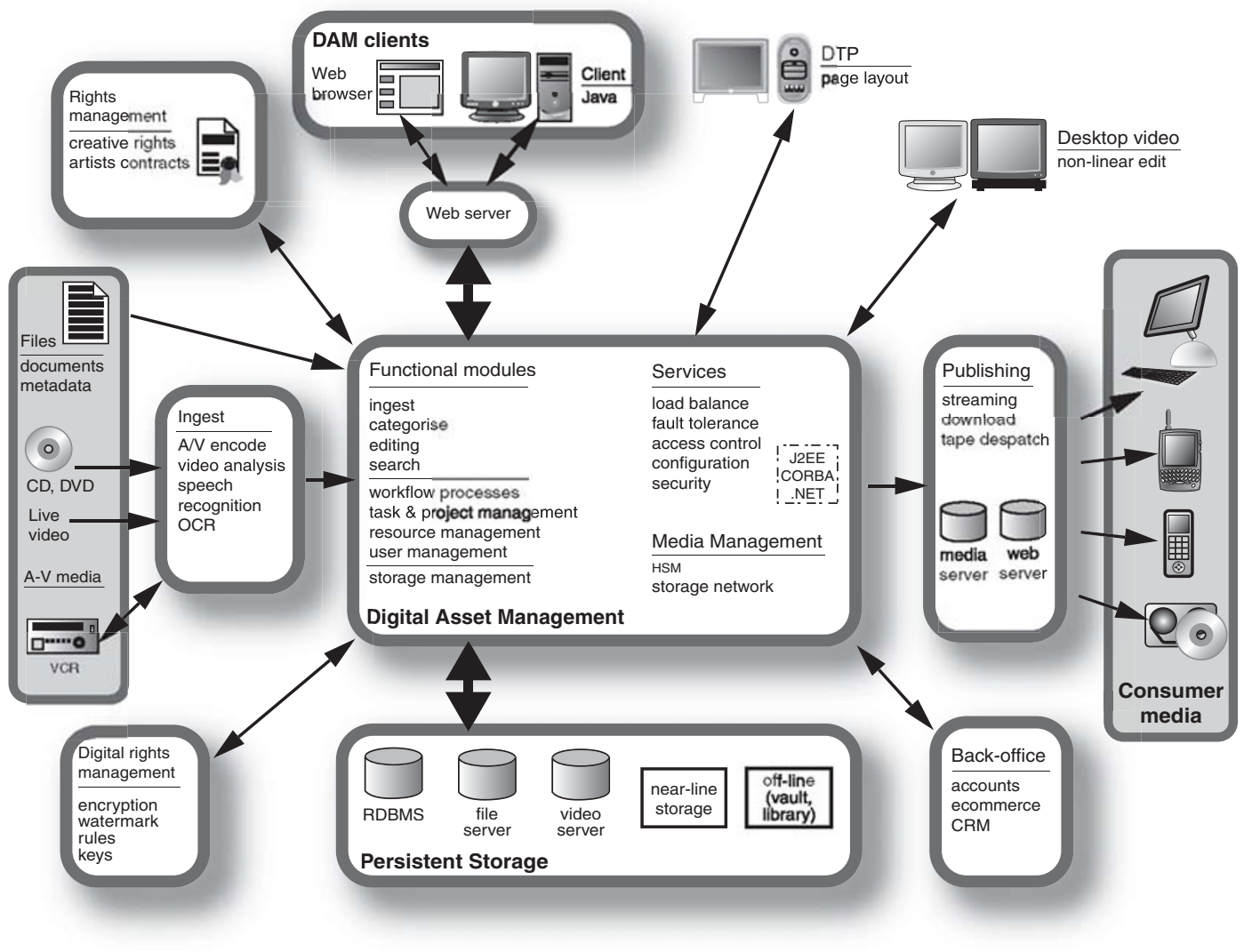

Figure 7: A typical DAM system 
management is potentially a single point of failure. A successful asset management system is going to become key to the efficient operation of the business, so high availability will be very important. The object broker platforms are usually designed to be distributed across a number of server devices. The load from the users is automatically balanced across the servers. In the event of device failure, the load can be rebalanced across the remaining facilities to give fault tolerance.

\section{Access control}

Access control is much like a database; users will be allocated a set of privileges to access folders, projects and files. These privileges may be rolebased as well as user-based. There may also be access to a project team or workgroup. The access control offered should be as flexible as possible to support different corporate structures.

\section{Security}

The content assets present an attractive target for unauthorized access or malicious damage. The security policies to protect the system should be much the same as the existing IT systems within the corporation. Security has to operate at many levels. Although access control will authorize users through the client portals of the asset management, it does not protect the persistent storage against direct access. The total security will include perimeter security, electronic and physical, operating system security, access control, and possibly encryption and watermarking of content within the repository.

\section{Configuration}

Just like any other large IT installation, the system will need constant configuration and maintenance.

\section{Peripherals}

Outside the core asset management application, there are a number of satellites. Some will be legacy systems, like rights management and the back-office systems. Others will be third-party applications that form part of the complete asset management.

\section{Web server}

This is the presentation layer of the DAM system. It is usually a conventional dynamic web server, much like a regular website. The web server creates the UI as an HTML page. It will offer the opportunity to brand the pages. This could be for external clients, or for use with departmental intranets. Audio-video previews may require additional specialized media servers to stream the proxy files. Although most users can connect to the system via the web server, craft workstations may connect directly to the application server.

\section{Ingest}

This is where some heavy number crunching takes place. For this reason it takes place on separate workstations. This is where the audiovideo content is parsed to make it searchable. The video is analyzed to create a storyboard of representative still frames. At the same time, a low-resolution copy can be made that can be used as a proxy of the content for preview purposes.

\section{Speech analysis}

The soundtrack of a video clip can be analyzed. With varying degrees of success, speech recognition software can extract the meaning of the spoken word. The resulting text transcript will be synchronized to the video and can be used to search and navigate through the clip. Some systems are also able to recognize different speakers and mark up the transcript accordingly.

\section{Scanning and optical character recognition}

Content such as paper documents can be scanned, and then read by optical character recognition (OCR) software. This will convert the content into a text file. OCR technology can also be used to read video graphics. An example of this would be the lower-third graphics used for television news broadcasts. These can be decoded and stored as textual data.

\section{File transfer}

Much content may already exist in a digital form. Files can be ingested by FTP or other file transfer from clients or partners. An ingest workstation can be used to register the content with the system and to add essential metadata: title, copyright owner, subject, and keywords. Some files may include comprehensive metadata; that can be ingested at the same time and used to populate the data tables. 


\section{Digital rights management}

Digital rights management (DRM) can be used to protect content from unauthorized access or viewing. It can be used to protect confidentiality or for the delivery of paid-for content. DAM can be used to manage the original encryption, setting the appropriate decryption rules to meet the business requirements.

\section{Publishing}

This will possibly be the most non-standard part of any system. The channels for the publishing of content may range from high-definition television and digital cinema, through web delivery of streaming media to the PC, all the way down to lightweight content for wireless applications. Fulfillment may also include physical media, CD-ROMs, DVDs or videotape.

\section{Third-party applications}

DAM can provide search, workflow and library facilities to many third-party applications within the enterprise.

\section{Video editing}

Non-linear editing is the primary application used for video post-production. The asset management can serve the editors as a repository for the raw elements of the production, and for the storage and archiving of finished material.

\section{Desktop publishing}

Many asset management products offer powerful links to Quark XPress, so that the desktop publisher can use the asset repository to store original text and graphics, as well as their finished work.

\section{Back-office}

There are many possible back-office applications that may be linked to the DAM system. For example, the workflow management could link to the accounts department to trigger the issue of invoices. The publishing side of the asset management may need links to e-commerce systems.

\section{Rights management}

This is the management of the contracts and agreements with content creators and owners. It is quite separate from digital rights. The rights are vital metadata for those involved in content creation and repurposing. It would prove very useful for the rights management to exchange metadata with the DAM.

\section{Persistent storage}

This is the main repository for content, as essence and metadata. The metadata are usually stored in a third-party relational database. Popular products include Oracle and Microsoft SQL Server. A regular file server can be used for content that can be stored in a standard file system like NFS, or NTFS. Such content may be documents and image files, or media files such as AVI, streaming media or QuickTime. Video is often stored on specialized video servers, but there are many developments that are allowing video - uncompressed, MPEG or DV - to be stored on normal file systems.

The persistent storage can be disk-based, or any combination of disk, data tape or optical storage. If removable storage is used, then media management will be needed to track the content. There are many possible architectures for the storage system. These include the storage networks (Storage Area Network and Network Attached Storage), hierarchical storage management (HSM), and the basic library systems used to manage tapes on shelves.

\section{Content strata}

Content is stored in strata to improve the efficiency of the overall system. Each stratum represents a layer of detail, with file sizes scaling appropriately:

- metadata;

- thumbnails;

- low-resolution preview;

- high resolution for production use.

A search can be made using small text files. The search can be refined using thumbnail images, perhaps a $50 \mathrm{kB}$ file. For video preview, a lowresolution proxy using one of the popular streaming formats gives efficient network utilization with data rates less than $1 \mathrm{Mb} / \mathrm{s}$. If the content is then required for editing or distribution then the very large high-resolution files can be accessed. 


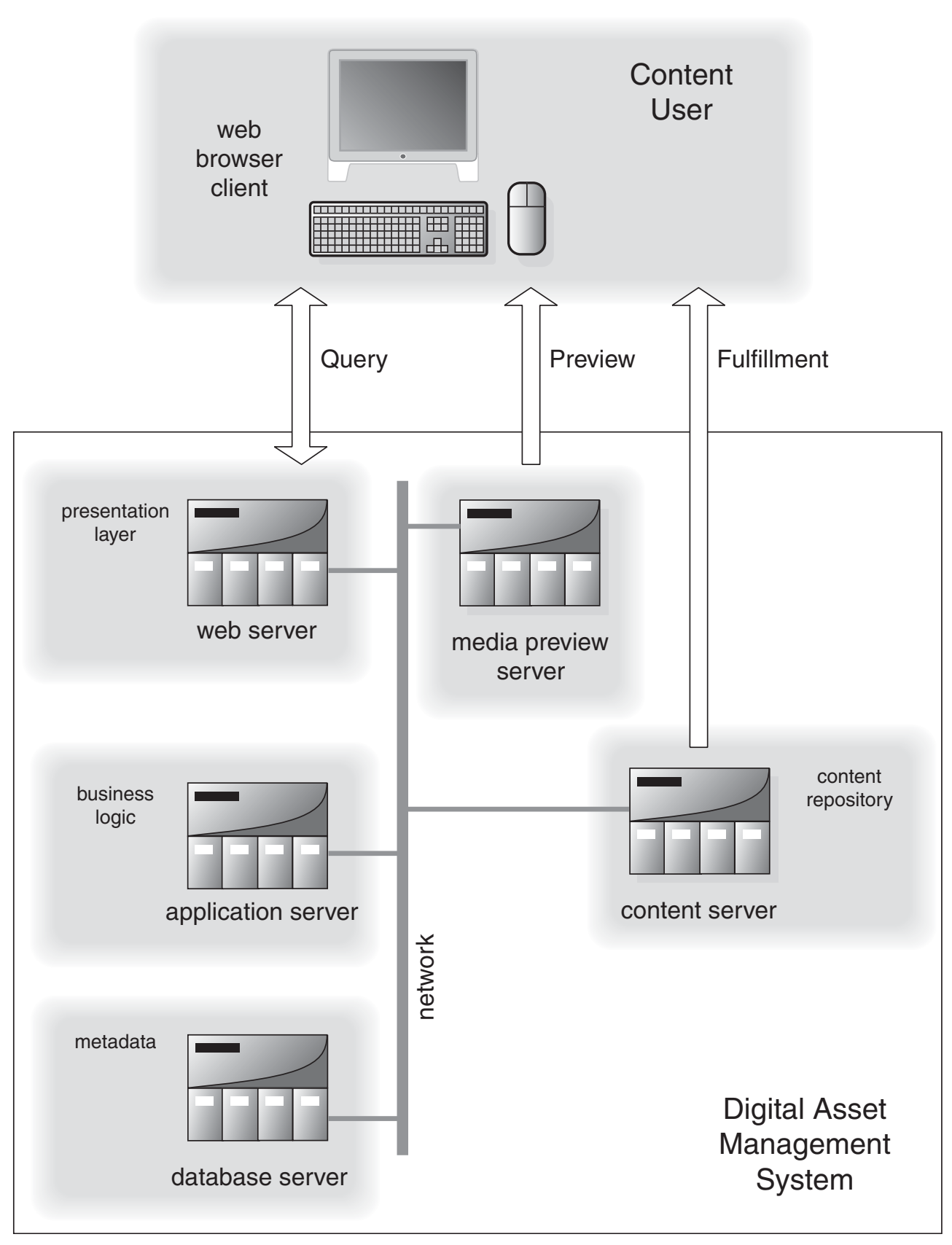

Figure 8: Clusters of servers

\section{Application platform}

The asset management system will run on a number of different server devices. Although it could all run on one machine, that would only be feasible for product demonstrations or small workgroups. To provide a system that can scale to support many users, the functions of the presentation layer, application server and database can be separated across different servers. These may even use different operating systems. The database could run on UNIX, the media server could run Windows Media services for streaming low-resolution video previews. 


\section{DISTRIBUTED COMPUTING}

The smallest asset management application may well be the single-user photo library. The application, database, and user interface are all rolled up into a single application. Put the photographer into a workgroup, and the application must become distributed. The requirement changes, in that the members of a workgroup want to share files and possibly collaborate on the editing of individual images.

The architecture changes to a workgroup server, and a number of clients that can view files on the server, and check out files for image processing. As the system scales up to large numbers of clients, the asset management database may well reach the limits of the integral database. To scale the system the data are broken out and stored on a separate relational database.

No database application is installed on the client; all data queries are performed by the asset management software on the workgroup server. When the client wants to make a query, the command is sent to the server, from where the database query is generated. The result set is then returned to the client. The client is making a remote call on the server to run a function or method; there is no need for the client to load the server application. This is the basis of distributed computing. Quite clearly, it has much in common with the multi-tier model.

In a large asset management system, a request for the metadata on a content file may require a query across a federation of different databases. The DAM database may store the core metadata, but other information could be held on a rights management system, or within the accounts department records. The asset management application server can make remote calls for reports from the other systems, then collate and format the information for return to the client. Note that the client does not have any accounting or rights management software loaded. The information is all retrieved through remote function calls.

The client can edit this information and return the dataset, and then the distributed databases are updated. This distribution is not without its problems. Traditional databases retain a persistent connection between the client and the database. The client can lock tables during data updates. The number of connections can be limited. It is not unknown for a potential user to phone round several co-workers to find one that could log-off the database in order to free up a connection.

Web clients are very different, in that they are stateless. A connection is made to download a page, and then it is closed. Persistent information about a client has to be maintained by the server using devices like cookies. There is an expectation of constant access to data, with no "busy tone." Since most users are not making constant access, the idea of effectively timesharing the connections makes sense.

The communication between the distributed applications is becoming more loosely coupled than the traditional real-time applications like transaction processing. This places a new set of demands upon the software architecture. The communication becomes based more on messages, almost a return to the days before the online database. This stateless messaging has other advantages. A fail-safe system can be put together. If one server fails, the client can simply communicate with another. So the system becomes truly distributed. This makes it very easy to scale a system to the point where it could be distributed across sites in different cities, or even to different continents. It is not without its drawbacks, one issue is the maintenance of data security - protecting the access to data stores to authorized users.

The development of distributed services is leaning towards the technology of XML web services. In this architecture each application media management, rights management, ecommerce - exposes functionality using internet protocols. To be specific, HTTP is used as a transport and XML as the data wrapper. Some vendors have started to adopt web services, and it promises to lower the costs of building complex distributed systems by using shrink wrap products for much of the infrastructure.

\section{SUMMARY}

Most asset management vendors have adopted the multi-tier model for their system architecture. The client can run a local application, or for basic search and preview functions, can be a normal web browser. The metadata are stored in a conventional relational database. The main asset management software runs on an application server. 
This architecture is very flexible; it can scale from a handful of users up to a large enterprise. It also simplifies software maintenance. The DBMS, web server and web clients are all very familiar products for the IT personnel to manage. The asset management software is typically object-oriented, using J2EE or CORBA, so with a suitable application server, can run on most operating systems, and will feature essential services like fail-over and loadbalancing that allows large and highly available systems to be assembled.

There is a talent pool of database administrators (DBAs) and web server administrators that can be called on to manage the bulk of a multi-tier system. Specialist staff are only required for the application server.

The asset management can be seen as the hub of a wheel. The spokes extend to third-party applications, either to share metadata, or to provide services to the asset management. The business logic integrates all these applications to give the user a federated view of media within the enterprise.

Distributed systems can scale to support a geographically dispersed business. Web services promise a new way to design the system architecture that offers scalable and robust systems, yet reduces the cost of ownership.

(C) David Austerberry 